\title{
3D models of Alzheimer's disease patient microglia recapitulate disease phenotype and show differential drug responses compared to 2D
}

\section{Carla Cuni-Lopez}

QIMR Berghofer Medical Research Institute https://orcid.org/0000-0001-8804-8970

Hazel Quek

QIMR Berghofer Medical Research Institute

Lotta Oikari

QIMR Berghofer Medical Research Institute

Romal Stewart

QIMR Berghofer Medical Research Institute

Tam Hong Nguyen

QIMR Berghofer Medical Research Institute

Yifan Sun

QIMR Berghofer Medical Research Institute

Christine Guo

QIMR Berghofer Medical Research Institute

Michelle Lupton

QIMR Berghofer Medical Research Institute

Anthony White ( $\square$ Tony.White@qimrberghofer.edu.au )

QIMR Berghofer Medical Research Institute

\section{Article}

Keywords: Alzheimer's disease (AD), 3D model, monocyte-derived microglia-like cells (MDMi)

Posted Date: July 29th, 2021

DOI: https://doi.org/10.21203/rs.3.rs-571254/v2

License: (c) (i) This work is licensed under a Creative Commons Attribution 4.0 International License.

Read Full License 


\section{Abstract}

Alzheimer's disease (AD) is an incurable neurodegenerative disorder with a rapidly increasing prevalence worldwide. Current approaches targeting hallmark pathological features of AD have had no consistent clinical benefit. Neuroinflammation is a major contributor to neurodegeneration and hence, microglia, the brain's resident immune cells, are an attractive target for potentially more effective therapeutic strategies. However, there is no current in vitro model system that faithfully recapitulates patient-specific microglial characteristics. To address this shortcoming, we developed novel 3D models of monocyte-derived microglia-like cells (MDMi) from AD patients. MDMi in 3D exhibited mature microglial features, including a highly branched morphology and enhanced bonafide microglial marker expression compared to $2 D$. AD MDMi in 3D co-culture with neuro-glial cells showed altered cell-to-cell interactions, growth factor and cytokine secretion profiles and responses to amyloid- $\beta$. Drug screening assays in 3D AD MDMi revealed different cytokine responses compared to 2D. Our study demonstrates disease- and drug-specific responses in 3D MDMi models that are not apparent in 2D and presents a new 3D platform for more effective and personalised drug testing.

\section{Introduction}

Alzheimer's disease (AD) is an age-related neurodegenerative disorder involving progressive impairment of cognitive functions. Although $A D$ - together with other neurodegenerative diseases - is predicted to become the second leading cause of death in the next 20 years, no prevention strategies or cure exist ${ }^{1}$. The most characteristic neuropathological hallmarks of $A D$ brains include extracellular deposits of misfolded amyloid- $\beta(A \beta)$ protein and intracellular neurofibrillary tangles of hyperphosphorylated tau protein. For decades, reducing protein aggregation has been the main therapeutic goal in $A D$, but this strategy has yielded poor clinical outcomes.

Chronic neuroinflammation is a critical component in the progression of many neurodegenerative diseases, including $A D^{2}$. Key neuroinflammatory effectors are microglia, the resident immune modulators of the brain. Microglial function is controlled by variants in AD risk genes (e.g., TREM2, APOE, CLU, CD33, $P I L R B$ and $P L C G 2)^{3}$. Such variants modulate microglial responses to $A D$ pathological events ${ }^{4-6}$, thus underlying the diversity of clinical phenotypes observed among patients. Hence, microglia represent a promising candidate for personalised, targeted therapeutics for AD.

Current $A D$ microglia in vitro model systems (reviewed in ${ }^{7,8}$ ) lack either clinical relevance or physiological complexity, thereby affecting translatability of drug outcomes into the clinic. For example, murine microglia lack the ability to fully recapitulate disease phenotype due to limited resemblance of immune functions and ageing processes between mice and humans ${ }^{9-12}$. Human immortalised microglia cell lines are genetically and functionally very different from in vivo microglia ${ }^{13-15}$. Post-mortem primary microglia isolated from $A D$ patients rapidly lose microglial signatures upon removal from the brain environment ${ }^{13}$. Conversely, human induced pluripotent stem cell (hiPSC)-derived microglia allow for the generation of a clinically relevant, patient-specific microglia platform. However, establishing hiPSC- 
derived microglia requires costly, long and technically challenging protocols that result in variable differentiation efficiencies ${ }^{16}$ and loss of patient-specific traits upon reprogramming ${ }^{17}$.

The monocyte-derived microglia-like cell (MDMi) model system addresses the shortcomings of the above models and provides a cost-effective approach for the rapid generation of personalised microglia cultures from living patients. This method has been previously applied by us and others using ex vivo bloodderived monocytes from schizophrenia ${ }^{18,19}$, Nasu-Hakola disease ${ }^{20}$ and amyotrophic lateral sclerosis (ALS) ${ }^{21}$ patients, demonstrating disease-associated phenotypes in patient-derived MDMi. In addition to their controlled genetic background, MDMi are readily available and yield mature microglia in a short time frame, thus allowing for the study of microglia from large patient cohorts ${ }^{22,23}$.

Microglial identity is driven by the multicellular milieu and three-dimensional (3D) network of macromolecules present in the brain. The lack of such structures in traditional two dimensional (2D) culture conditions greatly abrogate the ability of $2 \mathrm{D}$ in vitro models to replicate mature microglial function 24 . The use of 3D in vitro culture techniques and co-cultures with neuro-glial cells, which mimic the cues supporting microglial development in vivo, can increase the physiological relevance of the MDMi model. Indeed, an improved in vitro disease modelling capacity of 3D culture systems was demonstrated by the development of a complete $A D$ pathological cascade in $3 D$ but not in $2 D^{25,26}$. However, no $3 D$ in vitro model of $A D$ has yet incorporated patient-derived microglial cells in a highly reproducible and experimentally flexible $3 \mathrm{D}$ cell culture system ${ }^{27}$.

In this study, we generated for the first time 3D patient-specific MDMi models from living AD patients. These 3D hydrogel-based MDMi models are consistent and easy to generate and allow for the establishment of 3D MDMi co-cultures with human neuro-glial cells. We identified that 3D MDMi exhibit enhanced microglia-like features and AD-specific changes in the patient-derived models. To test the potential applicability of 3D MDMi platforms in a drug screening setting, we compared drug responses in MDMi between the 2D, 3D and 3D co-culture models. Together, the utility of the patient 3D MDMi models presented here opens new avenues for more predictable and personalised patient in vitro microglia models to test candidate therapeutics.

\section{Results}

\section{MDMi in 3D show increased survival and more mature microglial features compared to 2D}

We previously differentiated monocytes into MDMi in a 2D platform ${ }^{21}$. To develop a more physiologically relevant MDMi model, here we generated MDMi in a 3D platform. Monocytes embedded in Matrigel (Fig. 1A; Fig. S1A) resulted in cultures with 6.2-fold higher cell thickness (i.e., size of the Z-stack that captured the whole cell) compared to 2D (Fig. 1B; Fig. S1B; Movie S1, 2). Remarkably, MDMi survival in 3D was significantly increased by 2.5 fold compared to 2D (Fig. 1C). 
We next examined if the 3D culture affected morphology and expression of microglia-enriched markers in MDMi. 3D MDMi showed a highly ramified branched structure compared to 2D (Fig. 1D). Branched structure parameters -branch length and number of branches (endpoints) -, and branch complexity parameters -number of triple and quadruple junctions (points at which branches divide into three or four sub-branches, respectively) - were significantly increased in 3D compared to 2D MDMi (Fig. 1E-G). Ramified microglia have a highly branched morphology with a larger convex area than ameboid cells, thus correlating with a low ramification index ${ }^{28}$. The ramification index of 3D MDMi was lower than 2D MDMi (Fig. 1H), confirming an enhanced ramified morphology of MDMi in 3D likely due to the larger surface area for growth and differentiation provided by the 3D Matrigel scaffold.

Seminal microglial markers, including IBA1, PROS1, GPR34, TMEM119, GAS6 and TREM2, were upregulated and the leukocyte marker $C D 45$ was downregulated in 3D MDMi compared to monocytes (Fig. 1I). Interestingly, we observed a significantly increased expression of PROS1, GPR34 and TMEM119 in 3D MDMi compared to 2D (Fig. 1I). This suggests that the 3D platform promotes selective microgliaenriched markers in MDMi. As immature microglia have been reported to lack TMEM119 expression ${ }^{29}$, the upregulation of TMEM119 in 3D MDMi compared to 2D further indicates an enhanced microglial maturity in the 3D platform. Finally, positive immunostaining of Trem2 and P2ry 12 proteins in 3D MDMi confirmed the retention of microglial proteins in MDMi for up to 35 days (Fig. S1C).

\section{MDMi co-cultured in 3D with human neural progenitor cells elicit an inflammatory response to aggregated amyloid- $\beta(A \beta)$}

To mimic the neuro-glial cues of the brain microenvironment, we used the immortalised human neural progenitor cell (NPC) line ReNcell VM ${ }^{30}$ to establish a co-culture platform with MDMi.

Immunofluorescence and qRT-PCR marker characterisation showed that ReNcell VM lead to mixed populations of astrocytes, oligodendrocyte progenitor cells (OPCs) and neurons upon spontaneous differentiation while retaining a radial glial phenotype (Fig. S2A-C). Differentiation of 3D ReNcell VM cultures (Fig. S2D) demonstrated enhanced maturation of astrocytic and neuronal populations compared to $2 \mathrm{D}$ (Fig. SE, F), providing a basis for establishing a more mature co-culture platform of MDMi and neuroglia. For the 3D co-cultures, monocytes - pre-mixed with Matrigel - were added to 3D ReNcell VM and differentiated for 35 days, resulting in a mixed 3D co-culture containing MDMi and ReNcell VMderived neuro-glial cells (Fig. 2G). In 3D co-culture, monocytes readily differentiated into MDMi with a microglia-like morphology (Fig. 2H). In 2D co-culture, contrarily, monocytes retained their round morphology (Fig. S3), indicating that the support provided by the 3D Matrigel scaffold was necessary for co-culturing MDMi and ReNcell VM.

$A \beta$ aggregation is a major histopathological hallmark of $A D$ brains. Hence, we incorporated FITC-A $\beta$ peptides into 3D co-cultures. Remarkably, we observed that FITC-A $\beta$ peptides readily formed observable aggregates within $24 \mathrm{~h}$ of incubation in $3 \mathrm{D}$ (Fig. 2I) as opposed to 2D, where FITC-A $\beta$ remained unaggregated (Fig. S4). High $A \beta$ plaque load has been shown to induce pro-inflammatory responses in microglia from transgenic $A D$ mouse models ${ }^{31,32}$. Hence, we investigated whether MDMi are functional 
in $3 \mathrm{D}$ co-culture and respond to FITC-A $\beta$ aggregates. We observed a significantly upregulated secretion of classical pro-inflammatory cytokines IL-1 $\beta$ (2.8 fold) and IL-18 (2.3 fold), and similar trends for IL-6, in 3D co-cultures treated with FITC-A $\beta$ compared to untreated conditions (Fig. 2J). Secretion of other proinflammatory cytokines was also stimulated by FITC-A $\beta$ (Fig. S4). Such inflammatory responses to A $\beta$ were largely mediated by MDMi in the 3D co-cultures, as observed by significant cytokine secretion changes following treatment in 3D MDMi mono-cultures and no changes in 3D ReNcell VM monocultures (Fig. 2J, S5).

\section{Disease-specific differences in 2D and 3D mono-cultures of AD patient-derived MDMi}

We successfully generated 2D and 3D MDMi mono-cultures from healthy control $(\mathrm{HC})$ individuals and $A D$ patients (Fig. 3A) selected based on matched sex, age and APOE genotype (Table 1). Survival of both HC and AD MDMi was significantly increased in 3D compared to $2 \mathrm{D}$ by 2.6 and 2.4 fold, respectively (Fig. 3B).

Table 1

Summary of donor information.

\begin{tabular}{|llll|}
\hline Study cohorts & & Healthy Control (HC) & Alzheimer's disease (AD) \\
\hline No of participants & & $n=12$ & $n=13$ \\
\hline Sex of participants & Females (\%) & $58.3 \%(7 / 12)$ & $53.8 \%(7 / 13)$ \\
\cline { 2 - 4 } & Males (\%) & $41.7 \%(5 / 12)$ & $46.2 \%(6 / 13)$ \\
\hline Age of participants (mean \pm SD) & $68.5 \pm 2.7$ & $69.3 \pm 5.4$ \\
\hline APOE genotype & E3/E3 (\%) & $25 \%(3 / 12)$ & $7.7 \%(1 / 13)$ \\
\cline { 2 - 4 } & E3/E4 (\%) & $58.3 \%(7 / 12)$ & $69.2 \%(9 / 13)$ \\
\cline { 2 - 4 } & E4/E4 (\%) & $16.7 \%(2 / 12)$ & $23.1 \%(3 / 13)$ \\
\hline SD = standard deviation & & \\
\hline APOE = apolipoprotein E & & \\
\hline
\end{tabular}

Morphological examination of AD MDMi (Fig. S6) revealed that regardless of a 2D or 3D platform, HC and AD MDMi exhibit similar branch structure (branch length and number) (Fig. 3C, D) and complexity (number of triple and quadruple junctions, and ramification index) (Fig. 3E-G). Comparison of branched structure between 2D and 3D MDMi showed similar trends in HC and AD, including similar branch length and increased number of branches in 3D compared to 2D (Fig. 3C, D). Interestingly, this contrasted with branch length in a young $\mathrm{HC}$ cohort (20-40 years of age) of MDMi (Fig. 1E), where MDMi exhibited longer branches in 3D compared to $2 \mathrm{D}$.

Comparison of branch complexity between 2D and 3D MDMi showed an increased number of triple junctions in 3D compared to 2D MDMi in both HC and AD (Fig. 3E). Quadruple junctions increased in 3D 
compared to 2D MDMi in $\mathrm{HC}$ but remained unchanged in AD (Fig. 3F). Ramification index was significantly decreased in 3D compared to 2D MDMi in both $\mathrm{HC}$ and $\mathrm{AD}$ cohorts, confirming a more ramified morphology in 3D MDMi (Fig. 3G). Our results show that the 3D platform enhances branch number, ramified morphology and complexity parameters in MDMi from HC individuals compared to 2D. However, the 3D platform is limited in enhancing all complexity parameters in MDMi from AD patients compared to $2 \mathrm{D}$.

Next, we examined if $A D$ risk genes are differentially expressed in AD MDMi when cultured in 3D compared to 2D mono-cultures. A panel of AD risk genes, including CLU, TREM2, PLCG2 and PILRB, was examined by qRT-PCR. Fold change of expression in 3D compared to $2 \mathrm{D}$ revealed highly heterogeneous distributions within the $\mathrm{HC}$ and $\mathrm{AD}$ cohorts, with 3D MDMi showing trends of enhanced expression in $\mathrm{AD}$ patients compared to $\mathrm{HC}$ individuals for most risk genes (CLU, PLCG2 and PILRB) (Fig. $3 \mathrm{H}, \mathrm{I})$.

Interestingly, PILRB expression was significantly upregulated in AD 3D MDMi compared to HC (Fig. 3J), while no significant differences were observed for the other genes (Fig. S7). These results demonstrate that the 3D platform enhances the expression of microglia-specific AD risk genes in AD MDMi compared to $2 \mathrm{D}$ and reflects the heterogeneity of disease phenotypes within $A D$ patients.

\section{Disease-specific differences in 3D co-cultures of AD patient-derived MDMi}

We next characterised MDMi from $\mathrm{HC}$ individuals and $A D$ patients in the 3D co-culture platform (Fig. 4A). As observed in 3D MDMi mono-cultures, survival of MDMi in 3D co-culture was extended by 2.5 fold compared to 2D MDMi mono-cultures and was similar to 3D MDMi mono-cultures in both $\mathrm{HC}$ and $\mathrm{AD}$ cohorts (Fig. 4B). The increased survival of MDMi in 3D co-culture indicates that ReNcell VM do not affect MDMi viability. Consistently, similar expression of the apoptosis marker $B A X$ between $H C$ and $A D$ 3D co-cultures (Fig. 4C) suggests that the cell ratio of MDMi and ReNcell VM and the duration of the coculture were favourable.

To investigate whether AD MDMi reflect disease-specific differences in 3D co-culture, we analysed 1) cellto-cell interaction with neuro-glial cells, 2) secretion of growth factors and cytokines, and 3) migratory and inflammatory responses to $A \beta$ aggregates. The marked synapse loss in $A D$ is predominantly mediated by microglia through aberrant synapse engulfment ${ }^{33}$. Hence, we first examined if the cell-to-cell interactions between AD MDMi and ReNcell VM show differences compared to $\mathrm{HC}$ in the 3D co-cultures. $3 \mathrm{D}$ rendering and subsequent surface reconstruction of $3 \mathrm{D}$ co-culture images revealed a significantly smaller area of contact and a reduced number of contact points between MDMi and ReNcell VM in AD compared to HC 3D co-cultures (Fig. 4D-F). This suggests a possible impairment in the cell-to-cell interactions between MDMi and ReNcell VM in AD 3D co-cultures.

Microglia can secrete factors that alter neuron and astrocyte homeostasis, thereby contributing to $A D$ pathogenesis ${ }^{34}$. Hence, we compared the secretory profiles of $\mathrm{HC}$ and AD MDMi in 3D co-cultures after 35 days of co-culture. AD MDMi in 3D co-culture secreted higher levels of platelet-derived growth factor 
AA (PDGF-AA) and erythropoietin (EPO), and lower levels of interferon-y (IFN- $y)$, compared to HC in 3D coculture (Fig. 4G-I). This indicates that AD MDMi exhibit an altered secretory activity in the 3D co-culture. When comparing the secretion to 3D MDMi mono-cultures, we observed a significant upregulation of PDGF-AA, EPO (Fig. 4G, H) and other neurotrophic factors such as Angiopoietin 2 and the granulocytemacrophage colony stimulating factor (GM-CSF) (Fig. 4J, K) in 3D co-cultures from both $\mathrm{HC}$ and $\mathrm{AD}$ cohorts. Secretion of these factors by 3D ReNcell VM mono-cultures (dotted lines in Fig. 4G-K) changes compared to 3D MDMi mono-cultures, suggesting that the interaction between MDMi and ReNcell VM in the 3D co-cultures has a functional impact on either cell type.

Microglia in the vicinity of $A \beta$ plaques have been shown to exhibit altered proliferation, migration, clustering around $A \beta$ aggregates in a mouse model of $A D{ }^{35}$. Hence, in order to study MDMi behaviours in the presence of $A \beta$ depositions, we added FITC-A $\beta$ into HC and AD 3D co-cultures from and live-imaged for 7 days (Fig. 4L; Movie S3). AD MDMi surveyed longer distances and at a higher velocity around A $\beta$ aggregates compared to $\mathrm{HC}$, with no significant changes in the proportion of MDMi that clustered around the $A \beta$ aggregates (Fig. 4M-O). Interestingly, there were disease-specific differences in inflammatory cytokine secretion between $\mathrm{HC}$ and $A D$ MDMi in $3 D$ co-cultures treated with FITC-A $\beta$. When $A \beta$ was present, IL-6 secretion was significantly decreased in AD compared to HC 3D co-cultures, while increasing trends were observed for IL-1 $\beta$, IL-18 (Fig. 4P) and other pro-inflammatory cytokines such as TNF- $\alpha$ and IL-10 (Fig. S8). Such differences were not observed under untreated conditions. Together, these results indicate that $A D M D M i$ respond differently to AD-related stressors compared to $\mathrm{HC}$ when modelled in the 3D co-culture platform.

\section{Drug treatment induces differential cytokine gene expression in MDMi cultured in 2D and 3D}

Cytokines are key secreted molecules used by microglia to execute inflammatory and neuromodulatory functions and may have important roles in $A D$ pathogenesis ${ }^{36}$. To investigate cytokine expression profiles in MDMi we analysed a panel of inflammatory cytokines (IL-6, TNF- $a, I L-8, T G F-\beta, I L-10, I L-1 \beta$ and $I L-18)$ by qRT-PCR. When comparing cytokine expression levels between platforms, HC MDMi showed no significant differences (Fig. 5A) while in the AD cohort, 3D co-cultures showed a significant downregulation of $I L-8$ compared to 3D MDMi, and TGF- $\beta$ and $I L-18$ compared to 2D MDMi (Fig. 5B). Disease-specific differences were only observed in the 3D MDMi platform, where TNF- $a$ was downregulated in AD compared to HC (Fig. S9).

Based on the differences in baseline cytokine expression between platforms, we next investigated whether drug treatment alters such platform-dependent responses in the $\mathrm{HC}$ and $\mathrm{AD}$ cohorts. For this, we trialled two FDA-approved compounds - dasatinib and spiperone. These drugs have been shown to mitigate inflammation in in vitro models of microglia 37,38 and murine models of $A D^{39}$ and have potential as re-purposed drugs for treating neuroinflammation. Significant differences in pro-inflammatory cytokine responses to dasatinib treatment were most notable when comparing $3 \mathrm{D}$ to $2 \mathrm{D}$ MDMi mono-cultures, with $I L-6$ being downregulated in $\mathrm{HC}$ (Fig. 5C) and $I L-8, T G F-\beta$ and $I L-1 \beta$ being upregulated in AD 3D 
MDMi (Fig. 5D). Spiperone treatment only induced significant changes in TNF-a expression in the AD cohort (Fig. S10A, B). Interestingly, dasatinib modified cytokine expression levels compared to untreated conditions in the 2D platform but not in any of the 3D platforms (red triangles in Fig. 5C, D), suggesting potential implications on the capacity of cell model systems to predict drug outcomes in patients.

Interindividual variability in drug responses was displayed using heatmaps, which show cytokine expression levels in 2D and 3D MDMi mono-cultures from each individual of the $H C$ and $A D$ cohorts (Fig. 5E, F; Fig. S10C, D). Cytokine expression in HC and AD MDMi showed highly heterogeneous distributions in both 2D and 3D platforms. Cytokines that highly varied among individuals included IL-10 in $2 D$ and $I L-8$ in $3 D$ for both $H C$ and $A D$ (Fig. 5E, F). Interestingly, differences between dasatinib-treated 2D and 3D MDMi in specific HC or AD individuals were also evident, namely IL-8, IL-10 and IL-18 (Fig. 5E, F). Similar trends in the heterogeneity of drug responses were observed for spiperone-treated MDMi (Fig. S10C, D).

\section{Discussion}

Recreating physiologically relevant culture conditions to mimic the interaction of microglia with other brain cell types and the extracellular matrix is crucial for accurately modelling the role of microglia in disease. To date, no study has attempted to culture human non-genetically modified microglia in a 3D model system resembling human brain tissue. In an effort to develop more representative in vitro models of human microglia, we used MDMi - a model system of microglia-like cells that has emerged as a potential patient-specific drug screening platform for neurological diseases ${ }^{40,41}$. Here, we developed novel MDMi platforms that incorporate relevant in vivo cues resembling the microenvironment of the brain. Our study showed that a 3D hydrogel-based culture enhances MDMi survival, the extent and complexity of ramification (branching) and the expression of mature microglial markers (i.e., TMEM119) compared to standard 2D culture conditions. In addition, we have demonstrated the feasibility of culturing MDMi together with neuro-glial cells derived from human immortalised neural progenitor cells (i.e., ReNcell VM) in a 3D co-culture setting. Our findings also confirmed that MDMi can produce broad inflammatory responses upon exposure to $A \beta$, which is preserved in both $3 \mathrm{D}$ mono- and co-culture models.

Microglia are involved in the pathogenesis of $A D$ and contribute to the clinical heterogeneity observed among patients. 3D MDMi models offer a great opportunity to study AD microglia in a patient-specific manner. Unlike murine or human immortalised microglia cell lines used in previous studies ${ }^{42,43}, \mathrm{MDMi}$ are patient-derived and have not been genetically modified, being therefore more physiologically relevant and clinically applicable. In addition, the use of cell samples obtained from living patients allows for the longitudinal modelling of disease progression in $A D$, a prerequisite for targeted treatment at various stages of the disease. Analysis of MDMi branched morphology revealed similarities between $\mathrm{HC}$ and $A D$ $\mathrm{MDMi}$ in both the 2D and the 3D mono-culture models. These similarities most likely reflect age-related phenotypes described in human AD microglia rather than activated phenotypes observed in microglia from AD mouse models. Indeed, this age-related phenotype, termed HAM (human Alzheimer's microglia) 
was recently described in myeloid cell populations isolated from human AD post-mortem brain samples 44.

Modelling in 3D highlighted age- and disease-specific traits in MDMi morphology only evident when compared to 2D. Firstly, MDMi from elderly donors (60-80 years of age) failed to increase branch length in 3D as compared to MDMi from younger donors (20-40 years of age). This suggests that responses to branching signals and long-term culture in a 3D Matrigel scaffold are dependent on age. Secondly, MDMi from $A D$ patients failed to fully increase morphological complexity - in the form of junctions between branches - compared to 2D. Microglial branches are dynamic and constantly survey the brain microenvironment, phagocytosing dysfunctional synapses and releasing trophic factors that support neural connectivity ${ }^{45}$. Prior studies have identified a reduced number of junctions in human $A D$ microglial branches ${ }^{46}$ and less $A D$ microglial arborisation area compared to age-matched $\mathrm{HC}{ }^{47}$. Our findings highlight that despite differentiation in a brain-like 3D structural environment, $A D$ MDMi in 3D are not able to fully increase the outgrowth of sub-branches in the same way as HC MDMi.

Microglia establish physical contacts with neurons through identified molecular mechanisms ${ }^{48}$. Our results showed reduced cell-to-cell contacts between AD MDMi and ReNcell VM-derived neuro-glial cells compared to $\mathrm{HC}$ in the 3D co-cultures. Alterations in such microglia-neuron interactions could impact the microglial capacity to respond to neuronal damage, providing a potential mechanism underlying neuron degeneration in $A D$. Microglia have a canonical role in the removal of $A \beta$ aggregates ${ }^{49}$. We showed that AD MDMi migrate longer distances at a faster velocity compared to HC. Similarly, elevated migration rates were observed in human immortalised microglia cultured in a 3D tri-culture model with ReNcell VM overexpressing pathogenic $A \beta$ species ${ }^{42}$. However, an $A D$ mouse model with aberrant $A \beta$ production showed decreased microglial migration towards $A \beta$ plaques ${ }^{50}$, highlighting important differences between human and murine microglia responses to $A \beta$. Our findings warrant further investigation of microglial motility as a potentially dysregulated cellular feature in AD brains. Whether an increase in surveillance and velocity of AD MDMi correlates with an impaired branched complexity in these cells remains to be elucidated in future studies. Interestingly, we did not observe differences in the number of MDMi that clustered around $A \beta$ aggregates. However, we observed changes in pro-inflammatory cytokine secretion in MDMi from AD compared to $H C$. This suggests that AD MDMi may have unique diseasespecific chemotactic and secretory responses against $A \beta$. Future studies should investigate how such changes in MDMi impact the phagocytic clearance of $A \beta$ aggregates in the $3 D$ co-cultures. Overall, disease-specific differences exhibited by $A D M D M i$ in the 3D co-culture platform confirm the possibility to model disease in AD patient-specific MDMi using culture platforms that better recapitulate the brain microenvironment.

Preliminary drug testing demonstrated the utility of the 3D MDMi models as personalised drug screening tools. For instance, differences in MDMi drug responses between 2D and 3D culture conditions reflect the functional impact of MDMi cultured in a more biologically relevant 3D environment. This is in agreement with studies reporting more similarities in drug-induced cellular responses between 3D cultures and in 
vivo conditions than compared to $2 \mathrm{D}$ cultures ${ }^{51,52}$. Future investigations should determine whether drug responses from our 3D MDMi models correlate better with responses identified in animal models and clinical data from patients. Moreover, patient heterogeneity in MDMi drug responses was evident, supporting the translatability of our 3D platforms to measure individual patient responses in the clinic and further validating our in vitro systems as promising alternative platforms for personalised drug screening.

In future, the addition of patient hiPSC-derived neural progenitor cells into the 3D MDMi co-cultures would make this platform more personalised and likely a more accurate representation of the human brain. Similarly, using more-defined synthetic hydrogels - with more consistent and tuneable properties than Matrigel - may enable us to more carefully dissect the functionality of MDMi in 3D cultures.

In conclusion, we describe reproducible and easy-to-generate 3D in vitro models of MDMi that can recapitulate potentially important $A D$-specific differences - not identified in 2D models - associated with diseased microglia. This study opens new doors to generate patient-specific drug testing platforms that support the development of microglia-targeted therapeutic interventions tailored for AD patients and potentially other neurological disorders.

\section{Methods}

\section{Study cohort}

This study involved the recruitment of Alzheimer's disease (AD) and Healthy control (HC) participants through the Prospective Imaging Studying of Aging: Genes, Brain and Behaviour study (PISA) at QIMR Berghofer Medical Research Institute (QIMRB-MRI), Queensland, Australia ${ }^{53}$. All research adhered to ethical guidelines on human research outlined by the National Health and Medical Research Council of Australia (NHMRC) and ethical approval was obtained from QIMRB-MRI. All participants provided informed consent before participating in the study. The number of biological replicates varied in each assay due to the limited proliferative capacity of MDMi in culture, and the quantity of blood samples available from each donor. Further, repeated longitudinal sampling of peripheral blood from patients was not within the scope of this study. All samples used for assays were randomly selected, with matching age, gender and apolipoprotein E (APOE) status for each assay (Table 1). APOE genotyping was performed in the Sample Processing Facility at QIMRB-MRI, as previously described ${ }^{53}$.

\section{Isolation of peripheral blood mononuclear cells (PBMCs)}

Peripheral venous blood samples were collected in ethylenediaminetetraacetic acid (EDTA) tubes (BectonDickson, NJ, USA). PBMCs separation was performed within $2 \mathrm{~h}$ of blood withdrawal using SepMate ${ }^{\text {TM }}$ tubes (StemCell Technologies, BC, Canada) as per manufacturer's instructions. PBMCs were washed twice with PBS containing $1 \mathrm{mM}$ EDTA and subsequently frozen in $10 \%$ dimethyl sulphoxide (DMSO) (Merck KGaA, Hesse, Germany) and 90\% foetal bovine serum (ThermoFisher Scientific, CA, USA) (v/v). 


\section{Establishment of 2D and 3D MDMi cultures}

MDMi in 2D were generated as described previously ${ }^{21}$. Briefly, PBMCs were seeded onto Matrigel-coated plates (Corning, NY, USA). After $24 \mathrm{~h}$ incubation at $37^{\circ} \mathrm{C}, 5 \% \mathrm{CO}_{2}$, a cell population enriched in monocytes remained adhered to the culture vessel. Monocytes were then cultured in serum-free RPMI-1640 GlutaMAX medium (Life Technologies, Grand Island, NY, USA) supplemented with $0.1 \mu \mathrm{g} / \mathrm{ml}$ of interleukin (IL)-34 (IL-34) (Lonza, Basel-Stadt, Switzerland), $0.01 \mu \mathrm{g} / \mathrm{ml}$ of granulocyte-macrophage colonystimulating factor (GM-CSF) (Lonza) and 1\% (v/v) penicillin/streptomycin (Life Technologies) for 14 days. To induce MDMi differentiation in 3D, monocytes were resuspended in Matrigel diluted with ice-cold culture medium at a 1:3 ratio. Matrigel-cell mixtures were seeded in 96-well plates with medium containing $0.1 \mu \mathrm{g} / \mathrm{ml} \mathrm{IL-34}$ and $0.01 \mu \mathrm{g} / \mathrm{ml} \mathrm{GM-CSF}$. 3D MDMi were collected or used for downstream assays after 35 days in culture.

\section{Establishment of 2D and 3D human neural progenitor cell (NPCs) cultures}

The human ReNcell VM immortalised neural progenitor cell line (EMD Millipore, Billerica, MA, USA) was cultured as per manufacturer's instructions, with some modifications. Briefly, cells were plated onto Matrigel-coated plates for 2D cultures or mixed with a 1:3 Matrigel dilution to initiate the 3D cultures. ReNcell VM cultures were maintained in DMEM/F12 GlutaMAX medium (Life Technologies, Grand Island, NY, USA) containing $2 \%(\mathrm{v} / \mathrm{v}$ ) B27 supplement, $20 \mu \mathrm{g} / \mathrm{ml}$ epithelial growth factor (EGF) (Sigma-Aldrich, MO, USA), $20 \mu \mathrm{g} / \mathrm{ml}$ fibroblast growth factor 2 (FGF-2) (Lonza, Basel-Stadt, Switzerland) and 1\% (v/v) penicillin/streptomycin. Both 2D and 3D cultures were spontaneously differentiated by withdrawing growth factors from the maintenance medium (ReN base medium). All cells used were in passages 7-10 to ensure consistent spontaneous neuro-glial differentiation across independent experiments.

\section{Establishment of 3D co-cultures (MDMi and ReNcell VM)}

ReNcell VM were plated in 3D as described above and cultured for 1 day in ReN base medium to induce spontaneous differentiation. Monocytes were embedded in a 1:3 Matrigel dilution and seeded with 3D ReNcell VM cultures at 1:2.5 to 1:5 monocyte to ReNcell VM ratios. 3D co-cultures were maintained in $50 \%(\mathrm{v} / \mathrm{v})$ ReN base medium and MDMi culture medium for 35 days.

\section{Immunocytochemistry of 2D and 3D cultures}

Immunofluorescence staining of 2D and 3D cultures was performed as described previously ${ }^{21,54}$ with some modifications. 2D cultures were fixed in 4\% paraformaldehyde (PFA) or ice-cold methanol for 15 min and blocked at RT with $5 \%$ bovine serum albumin (BSA) (Sigma-Aldrich, MO, USA) in PBS. Primary antibodies (TREM 2 (1:500; Abcam, \# ab201621), P2RY12 (1:200; Alomone Labs, \#APR-20), TMEM119 (1:400; Abcam, \# ab185333), IBA1 (1:500; Wako, \#019-19741), Nestin (1:200; Abcam, \#ab22035), GFAP (1:2000; Abcam, \#ab4674), GalC (1:50; Santa Cruz, \#sc-518055), Doublecortin (DCX) (1:200; Abcam, ab36447) and $\beta$ III-tubulin (TUBB3) (1:500; BioLegend, \#801202)) were diluted in blocking solution and incubated overnight at $4^{\circ} \mathrm{C}$. Cells were then washed three times with $0.1 \%$ Triton-X 100 in PBS and 
incubated with secondary antibodies (1:250 Alexa Fluor 488 (\#A-11034) / 594 (\#A-21203) / 647 (\#A21244) (ThermoFisher Scientific, CA, USA)) for $2 \mathrm{~h}$ at RT in the dark and counterstained with a nuclear dye (Hoechst 33342, $1 \mu \mathrm{g} / \mathrm{ml}$ ). 3D cultures were fixed with 4\% PFA overnight at RT, permeabilised for 30 min with $0.3 \%$ Triton-X 100 in PBS, and blocked overnight at $4^{\circ} \mathrm{C}$ with $2 \%$ BSA (Sigma-Aldrich, MO, USA) in PBS. Primary antibody solutions were incubated for $24 \mathrm{~h}$ at $4^{\circ} \mathrm{C}$. Secondary antibody solutions were incubated for $5 \mathrm{~h}$ at $4^{\circ} \mathrm{C}$ in the dark. Cultures were then washed five times (10 min each) with $0.1 \%$ Triton$X 100$ in PBS and counterstained with Hoechst 33342. Images were captured using a confocal laser scanning microscope (LSM-780, Carl Zeiss) at 20X and 40X magnification and processed using the Zeiss ZEN software.

\section{RNA extraction and quantitative real-time PCR (qRT-PCR)}

RNA and cDNA were prepared as previously described ${ }^{55}$. Total RNA was extracted using a Direct-zol RNA Miniprep kit (Integrated Sciences, Australia) as per manufacturer's protocol. Conversion to cDNA was carried out using a SensiFAST ${ }^{\mathrm{TM}}$ cDNA synthesis kit (Bioline, London, UK). For qRT-PCR, cDNA was diluted 1:10 to generate working solutions and combined with SensiFAST ${ }^{\mathrm{TM}}$ SYBR ${ }^{\circledR}$ Lo-ROX master mix and gene-specific primers. qRT-PCR runs were performed as triplicate on Applied Biosystems ViiA 7 (ThermoFisher Scientific, CA, USA). Endogenous control 18S was used as a housekeeping gene for normalisation. Relative gene expression levels were calculated using the $\Delta \Delta \mathrm{Ct}$ method. Human primer sequences used are listed in Supplementary Table S1.

\section{Multiplex bead-based immunoassay}

The LEGENDplexTM Human Inflammation (\#740809) and Growth Factor (\#740180) kits (BioLegend, CA, USA) were used to detect cytokines and growth factors in conditioned media. The assay was performed as per manufacturer's instructions. Briefly, conditioned media were incubated with a cocktail of antibodyconjugated capture beads. Then biotinylated detection antibodies were added followed by streptavidinphycoerythrin (SA-PE). The amount of analytes of interest in the samples was calculated as a proportion of the fluorescent signal intensity provided by capture bead-analyte-detection antibody-SA-PE sandwiches. Signals were acquired on a BD LSRFortessa 5 (BD Biosciences, CA, USA) using FACSDiva software, and analysed using Qognit, a cloud-based LEGENDplexTM software (BioLegend, CA, USA). Concentrations $(\mathrm{pg} / \mathrm{ml})$ were normalised to total amount of protein in the cultures.

\section{Morphology analysis}

Quantification of morphological parameters of MDMi was performed by adapting a previous method ${ }^{56}$. Phase contrast images acquired with a 20X objective were processed in FIJI software (National Institutes of Health, Maryland, USA) using a macro script that applied a threshold, followed by processing functions "despeckle", "close" and "remove outliers" that generated a binary image. Binary images were then run on the AnalyseSkeleton(2D/3D) plugin, which resulted in skeletonised images. The "results and branch information" outputs from the plugin contained data on branch length, endpoint number and triple and quadruple junctions number. Binary images were also analysed using the Analyze particles function in FIJI. This calculated the "solidity" or "ramification index" value, which results from dividing the area of 
MDMi by its convex area (i.e., area of the smallest polygon drawn around the cell). More ramified cells have a bigger convex area and thus a smaller ramification index $(<1)$. Mean single cell values for each parameter were calculated. The total number of MDMi analysed per donor was 100 in 2D and 20 in 3D.

\section{Cell contacts analysis in 3D co-cultures}

Confocal Z-stack images of 3D co-cultures acquired with a 20X objective were rendered in 3D using the Imaris software (Bitplane, Belfast, UK) and analysed with the Surface-Surface contact area extension module. During image acquisition, the Z-interval was set at "Optimal" so that the number of acquired slices was suitable for the given stack size, objective lens, and pinhole diameter. Following surface modelling using the Surface function in Imaris, the Surface-Surface contact area extension module was applied to measure the areas in contact between ReNcell VM and MDMi as well as the number of contacts established. Both parameters were then normalised to the total number of MDMi in the image. A total of $200 \mathrm{MDMi}$ in co-culture were analysed for each donor in the $\mathrm{HC}$ and $\mathrm{AD}$ cohorts.

\section{Preparation of amyloid- $\beta(A \beta)$ aggregates}

Fluorescein isothiocyanate (FITC)-conjugated amyloid- $\beta$ peptides 1-42 (FITC-A 1 1-42) (Bachem, M2585, $\mathrm{CH}$ ) were dissolved in DMSO to a concentration of $500 \mu \mathrm{M}$ and stored at $-80^{\circ} \mathrm{C}$. FITC-A $\beta 1-42$ were incubated for $24 \mathrm{~h}$ at $37^{\circ} \mathrm{C}$ in the $3 \mathrm{D}$ cultures prior to imaging to allow for the fibrillisation of the peptides and formation of $A \beta$ aggregates.

\section{$A \beta$ aggregates exposure and surveillance analysis}

FITC-A $1-42$ peptides were added at $5 \mu \mathrm{M}$ to MDMi 3D mono- and co-cultures at day 35 of differentiation. After $24 \mathrm{~h}$, cultures were imaged on an EVOS FL Auto 2 (ThermoFisher Scientific, CA, USA). Scans were set to image multiple z-stack planes every $12 \mathrm{~h}$ for 7 days using a $10 \mathrm{X}$ objective. At least 3 fields of view were scanned per well. MDMi located within an area of 90,000 $\mu \mathrm{m} 2$ containing one or more $A \beta$ aggregate were tracked using the Manual tracking plugin in FIJI. Migrated distance and velocity of tracked cells were calculated and normalised to the number of MDMi in the analysed areas. Between 100-200 MDMi per individual were tracked in both HC and AD cohorts.

\section{Statistical analysis}

All statistical analyses were performed using GraphPad Prism software version 8 (Graphpad Software, CA, USA). Comparisons between two groups were analysed with two-tailed Student's t-test or MannWhitney $\mathrm{U}$, when normality assumptions were not met. Comparisons between three or more groups were analysed by one- or two-way analysis of variance (ANOVA) followed by post-hoc tests. Data are presented as mean \pm SEM or mean $\pm S D$ and $P \leq 0.05$ was considered significant. Statistical significance was determined as ${ }^{*} P<0.05,{ }^{*} \mathrm{P}<0.01,{ }^{* \star *} \mathrm{P}<0.001,{ }^{* \star *} \mathrm{P}<0.0001$, as detailed in figure legends.

\section{Declarations}

Acknowledgements 
We would like to thank the volunteers from QIMR Berghofer who have donated blood to this project and the participants recruited through the PISA study. We also thank the Microscopy, Flow cytometry, Sample Processing and Statistical teams at QIMR Berghofer for their assistance. Special thanks to Kurt Giulani

for helpful insights on LEGENDplex ${ }^{T M}$ data analysis. Lastly, we acknowledge the coordinators of the PISA study, Professor Michael Breakspear, Jessica Adsett and Natalie Garden.

\section{Author contributions}

C.C-L., H.Q., L.E.O. and A.R.W. conceived and designed the study. C.C-L. and H.Q. performed experiments. C.C-L., H.Q. and T.H.N. analysed data. C.C-L., H.Q., R.S. and A.R.W. interpreted data. Y.S., C.C.G. and M.K.L. coordinated blood collection from the PISA study. C.C-L. wrote the manuscript. H.Q., R.S., L.E.O. and A.R.W. provided critical feedback in reviewing and editing. All authors reviewed and commented on the final version of the manuscript.

\section{Competing interests}

A.R.W. and H.Q. are listed as co-authors of provisional patent AU2020/050513. The authors declare no other competing interests.

\section{References}

1. Gammon, K. Neurodegenerative disease: brain windfall. Nature 515, 299-300, doi:10.1038/nj7526299a (2014).

2. Heneka, M. T. et al. Neuroinflammation in Alzheimer's disease. Lancet Neurol. 14, 388-405 (2015).

3. Jack Jr, C. R. et al. Hypothetical model of dynamic biomarkers of the Alzheimer's pathological cascade. Lancet Neurol. 9, 119-128 (2010).

4. De Strooper, B. \& Karran, E. The cellular phase of Alzheimer's disease. Cell 164, 603-615 (2016).

5. Sala Frigerio, C. et al. The Major Risk Factors for Alzheimer's Disease: Age, Sex, and Genes Modulate the Microglia Response to Aß Plaques. Cell Reports 27, 1293-1306.e1296, doi:10.1016/j.celrep.2019.03.099 (2019).

6. Sierksma, A. et al. Novel Alzheimer risk genes determine the microglia response to amyloid- $\beta$ but not to TAU pathology. EMBO Mol. Med. 12, e10606 (2020).

7. Stansley, B., Post, J. \& Hensley, K. A comparative review of cell culture systems for the study of microglial biology in Alzheimer's disease. J. Neuroinflammation 9, 1-8 (2012).

8. Haenseler, W. \& Rajendran, L. Concise Review: Modeling Neurodegenerative Diseases with Human Pluripotent Stem Cell-Derived Microglia. Stem Cells 37, 724-730 (2019).

9. Holtman, I. R. et al. Induction of a common microglia gene expression signature by aging and neurodegenerative conditions: a co-expression meta-analysis. Acta neuropathologica communications 3, 31-31, doi:10.1186/s40478-015-0203-5 (2015). 
10. Galatro, T. F. et al. Transcriptomic analysis of purified human cortical microglia reveals ageassociated changes. Nat. Neurosci. 20, 1162-1171, doi:10.1038/nn.4597 (2017).

11. Gosselin, D. et al. An environment-dependent transcriptional network specifies human microglia identity. Science 356, eaal3222, doi:10.1126/science.aal3222 (2017).

12. Smith, A. M. \& Dragunow, M. The human side of microglia. Trends Neurosci. 37, 125-135, doi:https://doi.org/10.1016/j.tins.2013.12.001 (2014).

13. Butovsky, 0 . et al. Identification of a unique TGF- $\beta$-dependent molecular and functional signature in microglia. Nat. Neurosci. 17, 131-143, doi:10.1038/nn.3599 (2014).

14. Das, A. et al. Transcriptome sequencing reveals that LPS-triggered transcriptional responses in established microglia BV2 cell lines are poorly representative of primary microglia. $J$. Neuroinflammation 13, 1-18 (2016).

15. Melief, J. et al. Characterizing primary human microglia: A comparative study with myeloid subsets and culture models. Glia 64, 1857-1868 (2016).

16. Sabogal-Guáqueta, A. M. et al. Microglia alterations in neurodegenerative diseases and their modeling with human induced pluripotent stem cell and other platforms. Prog. Neurobiol. 190, 101805, doi:https://doi.org/10.1016/j.pneurobio.2020.101805 (2020).

17. Mertens, J., Reid, D., Lau, S., Kim, Y. \& Gage, F. H. Aging in a Dish: iPSC-Derived and Directly Induced Neurons for Studying Brain Aging and Age-Related Neurodegenerative Diseases. Annu. Rev. Genet. 52, 271-293, doi:10.1146/annurev-genet-120417-031534 (2018).

18. Sellgren, C. M. et al. Increased synapse elimination by microglia in schizophrenia patient-derived models of synaptic pruning. Nature Neuroscience 22, 374-385, doi:10.1038/s41593-018-0334-7 (2019).

19. Ormel, P. R. et al. A characterization of the molecular phenotype and inflammatory response of schizophrenia patient-derived microglia-like cells. Brain, Behav., Immun. 90, 196-207, doi:https://doi.org/10.1016/j.bbi.2020.08.012 (2020).

20. Ohgidani, M. et al. Direct induction of ramified microglia-like cells from human monocytes: Dynamic microglial dysfunction in Nasu-Hakola disease. Sci. Rep. 4, 4957, doi:10.1038/srep04957 (2014).

21. Quek, H. et al. ALS monocyte-derived microglia reveal cytoplasmic TDP-43 accumulation, DNA damage, and cell-specific impairment of phagocytosis associated with disease progression. bioRxiv, 2020.2010.2025.354399, doi:10.1101/2020.10.25.354399 (2020).

22. Ryan, K. J. et al. A human microglia-like cellular model for assessing the effects of neurodegenerative disease gene variants. Sci. Transl. Med. 9, eaai7635, doi:10.1126/scitranslmed.aai7635 (2017).

23. Penney, J., Ralvenius, W. T. \& Tsai, L.-H. Modeling Alzheimer's disease with iPSC-derived brain cells. Mol. Psychiatry 25, 148-167, doi:10.1038/s41380-019-0468-3 (2020).

24. Watson, P. M. D., Kavanagh, E., Allenby, G. \& Vassey, M. Bioengineered 3D glial cell culture systems and applications for neurodegeneration and neuroinflammation. SLAS Discov. 22, 583-601 (2017). 
25. Choi, S. H. et al. A three-dimensional human neural cell culture model of Alzheimer's disease. Nature 515, 274-278, doi:10.1038/nature13800 (2014).

26. D'avanzo, C. et al. Alzheimer's in 3D culture: challenges and perspectives. Bioessays 37, 1139-1148 (2015).

27. Hedegaard, A., Stodolak, S., James, W. S. \& Cowley, S. A. Honing the Double-Edged Sword: Improving Human iPSC-Microglia Models. Front. Immunol. 11, doi:10.3389/fimmu.2020.614972 (2020).

28. Zanier, E. R., Fumagalli, S., Perego, C., Pischiutta, F. \& De Simoni, M.-G. Shape descriptors of the "never resting" microglia in three different acute brain injury models in mice. Intensive Care Med Exp 3, 39-39, doi:10.1186/s40635-015-0039-0 (2015).

29. Satoh, J.-i. et al. TMEM119 marks a subset of microglia in the human brain. Neuropathology 36, 3949, doi:https://doi.org/10.1111/neup.12235 (2016).

30. Donato, R. et al. Differential development of neuronal physiological responsiveness in two human neural stem cell lines. BMC Neurosci. 8, 36, doi:10.1186/1471-2202-8-36 (2007).

31. Frautschy, S. A. et al. Microglial response to amyloid plaques in APPsw transgenic mice. Am. J. Pathol. 152, 307-317 (1998).

32. Stalder, M. et al. Association of microglia with amyloid plaques in brains of APP23 transgenic mice. Am. J. Pathol. 154, 1673-1684, doi:10.1016/S0002-9440(10)65423-5 (1999).

33. Rajendran, L. \& Paolicelli, R. C. Microglia-mediated synapse loss in Alzheimer's disease. J. Neurosci. 38, 2911-2919 (2018).

34. Colonna, M. \& Butovsky, O. Microglia Function in the Central Nervous System During Health and Neurodegeneration. Annu. Rev. Immunol. 35, 441-468, doi:10.1146/annurev-immunol-051116052358 (2017).

35. Zhong, L. et al. Soluble TREM2 ameliorates pathological phenotypes by modulating microglial functions in an Alzheimer's disease model. Nat. Commun. 10, 1365, doi:10.1038/s41467-019-091189 (2019).

36. Zheng, C., Zhou, X.-W. \& Wang, J.-Z. The dual roles of cytokines in Alzheimer's disease: update on interleukins, TNF- $\alpha$, TGF- $\beta$ and IFN-y. Transl. Neurodegener. 5, 7, doi:10.1186/s40035-016-0054-4 (2016).

37. Ryu, K.-Y. et al. Dasatinib regulates LPS-induced microglial and astrocytic neuroinflammatory responses by inhibiting AKT/STAT3 signaling. J. Neuroinflammation 16, 190, doi:10.1186/s12974019-1561-x (2019).

38. Zheng, L. T. et al. The antipsychotic spiperone attenuates inflammatory response in cultured microglia via the reduction of proinflammatory cytokine expression and nitric oxide production. $J$. Neurochem. 107, 1225-1235, doi:10.1111/j.1471-4159.2008.05675.x (2008).

39. Dhawan, G. \& Combs, C. K. Inhibition of Src kinase activity attenuates amyloid associated microgliosis in a murine model of Alzheimer's disease. J. Neuroinflammation 9, 117, doi:10.1186/1742-2094-9-117 (2012). 
40. King, A. The search for better animal models of Alzheimer's disease. Nature 559, S13-S13 (2018).

41. Ohgidani, M., Kato, T. A. \& Kanba, S. Introducing directly induced microglia-like (iMG) cells from fresh human monocytes: a novel translational research tool for psychiatric disorders. Front. Cell. Neurosci. 9, 184 (2015).

42. Park, J. et al. A 3D human triculture system modeling neurodegeneration and neuroinflammation in Alzheimer's disease. Nat. Neurosci. 21, 941-951, doi:10.1038/s41593-018-0175-4 (2018).

43. Haw, R. T. Y. et al. A three-dimensional collagen construct to model lipopolysaccharide-induced activation of BV2 microglia. J. Neuroinflammation 11, 134, doi:10.1186/1742-2094-11-134 (2014).

44. Srinivasan, K. et al. Alzheimer's patient microglia exhibit enhanced aging and unique transcriptional activation. Cell Rep. 31, 107843 (2020).

45. Nimmerjahn, A., Kirchhoff, F. \& Helmchen, F. Resting microglial cells are highly dynamic surveillants of brain parenchyma in vivo. Science 308, 1314-1318 (2005).

46. Paasila, P. J., Davies, D. S., Kril, J. J., Goldsbury, C. \& Sutherland, G. T. The relationship between the morphological subtypes of microglia and Alzheimer's disease neuropathology. Brain Pathology 29, 726-740, doi:10.1111/bpa.12717 (2019).

47. Davies, D. S., Ma, J., Jegathees, T. \& Goldsbury, C. Microglia show altered morphology and reduced arborization in human brain during aging and A Izheimer's disease. Brain Pathol. 27, 795-808 (2017).

48. Cserép, C. et al. Microglia monitor and protect neuronal function through specialized somatic purinergic junctions. Science 367, 528-537 (2020).

49. Lai, A. Y. \& McLaurin, J. Clearance of amyloid- $\beta$ peptides by microglia and macrophages: the issue of what, when and where. Future Neurol. 7, 165-176, doi:10.2217/fnl.12.6 (2012).

50. Bolmont, T. et al. Dynamics of the microglial/amyloid interaction indicate a role in plaque maintenance. J. Neurosci. 28, 4283-4292, doi:10.1523/JNEUROSCI.4814-07.2008 (2008).

51. Zietarska, M. et al. Molecular description of a 3D in vitro model for the study of epithelial ovarian cancer (EOC). Mol. Carcinog. 46, 872-885, doi:https://doi.org/10.1002/mc.20315 (2007).

52. Souza, A. G. et al. Comparative assay of 2D and 3D cell culture models: proliferation, gene expression and anticancer drug response. Curr. Pharm. Des. 24, 1689-1694 (2018).

53. Lupton, M. K. et al. A prospective cohort study of prodromal Alzheimer's disease: Prospective Imaging Study of Ageing: Genes, Brain and Behaviour (PISA). Neuroimage Clin. 29, 102527, doi:https://doi.org/10.1016/j.nicl.2020.102527 (2021).

54. Kim, Y. H. et al. A 3D human neural cell culture system for modeling Alzheimer's disease. Nat. Protoc. 10, 985-1006, doi:10.1038/nprot.2015.065 (2015).

55. Oikari, L. E. et al. Altered Brain Endothelial Cell Phenotype from a Familial Alzheimer Mutation and Its Potential Implications for Amyloid Clearance and Drug Delivery. Stem Cell Reports 14, 924-939, doi:https://doi.org/10.1016/j.stemcr.2020.03.011 (2020). 
56. Young, K. \& Morrison, H. Quantifying Microglia Morphology from Photomicrographs of Immunohistochemistry Prepared Tissue Using ImageJ. J Vis Exp, 57648, doi:10.3791/57648 (2018).

\section{Figures}

A

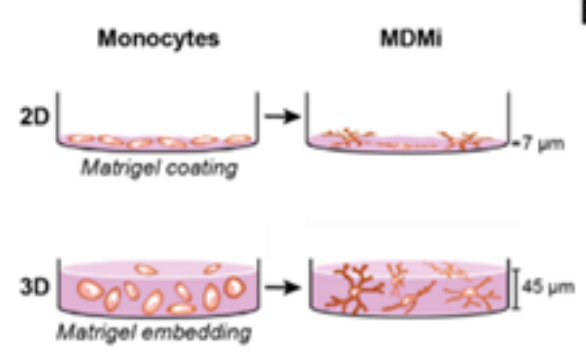

B

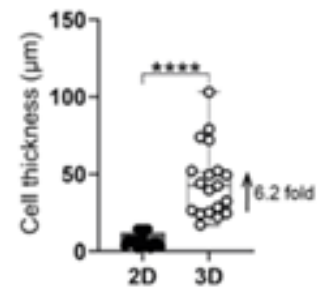

C

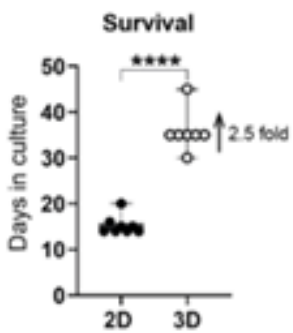

D

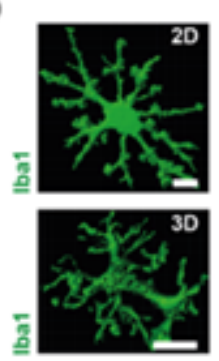

H

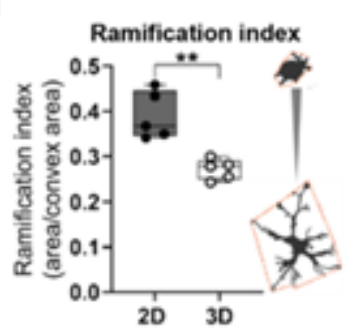

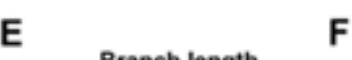

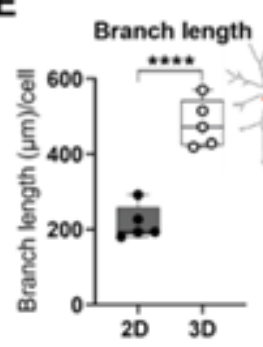

$\mathbf{F}$

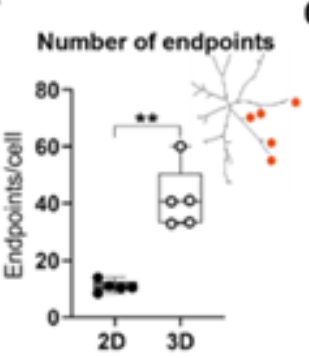

G

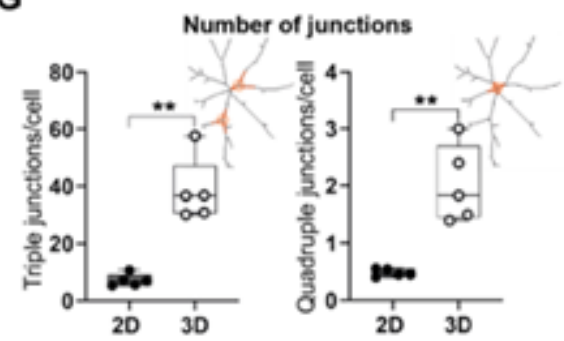

I

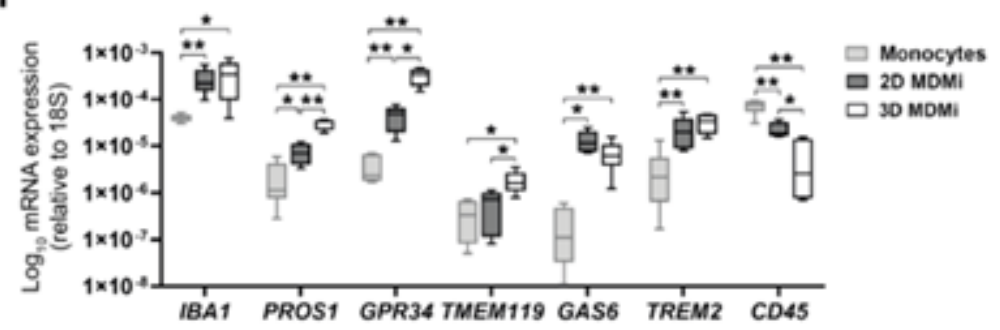

\section{Figure 1}

Generation and characterisation of distinctive microglia features in 2D and 3D MDMi. (A) Schematic illustration of monocyte differentiation into MDMi in 2D, achieved by seeding the monocytes on a Matrigel-coated surface, or in 3D, achieved by embedding the monocytes in a thick Matrigel layer. (B) Cell thickness of MDMi in 2D ( $n=17$ independent cultures) and 3D ( $n=19$ independent cultures) cultures. (C) Survival of 2D ( $n=8)$ and 3D MDMi $(n=7)$. (D) 3D surface rendered images of 2D and 3D MDMi stained for Iba1 (green). Scale bars, $25 \mu \mathrm{m}$. See also Supplementary Movies S1 and S2. Quantification of morphological parameters in 2D $(n=5)$ and 3D MDMi $(n=5)$, including $(E)$ branch length, $(F)$ number of endpoints, $(G)$ number of junctions, including triple junctions (left) and quadruple junctions (right) and $(\mathrm{H})$ ramification index (area/convex area). Representative skeleton and binary images are included on the right of each graph to illustrate the morphological measurements. (I) mRNA expression of microglia(IBA1, PROS1, GPR34, TMEM119, GAS6, TREM2) and leukocyte-enriched (CD45) markers in the starting monocyte population $(n=6)$ and the resulting 2D $(n=7)$ and 3D $(n=6)$ MDMi cultures. Data are presented as mean \pm SD. Each single data point represents one biological replicate. Unpaired Student's $t$ 
test with or without Welch's correction, two-tailed; one-way ANOVA with Tukey's multiple comparison test in $\mathrm{I} ; * \mathrm{P}<0.05, * * \mathrm{P}<0.01, * \star \star * \mathrm{P}<0.0001$.

A

3D co-culture

Day 0

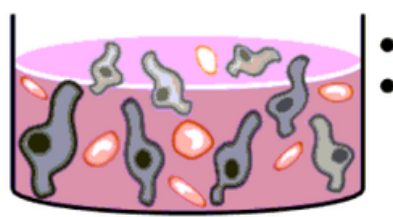

Matrigel embedding
3D co-culture

Day 35
B

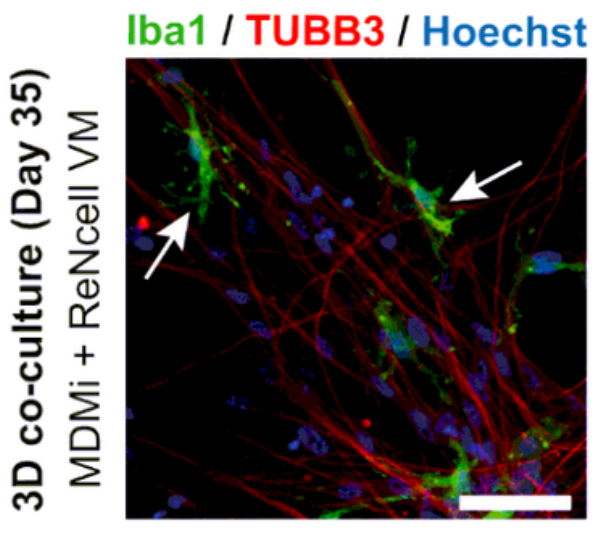

- Monocytes

ReNcell VM

(Day 1 spontaneous differentiation)

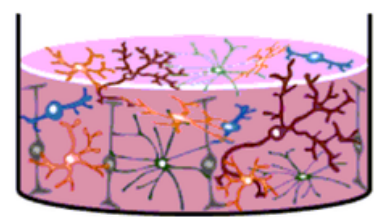

MDMi

ReNcell VM-derived:

Radial glia

Neurons

Astrocytes

OPCs

C

\section{Iba1 / GFAP / Hoechst}

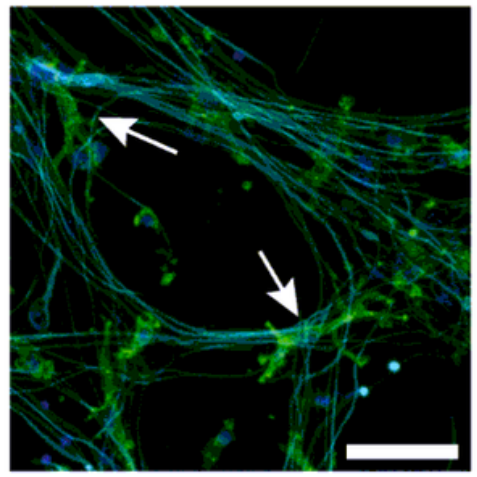

Iba1 / TUBB3 / A $\beta$

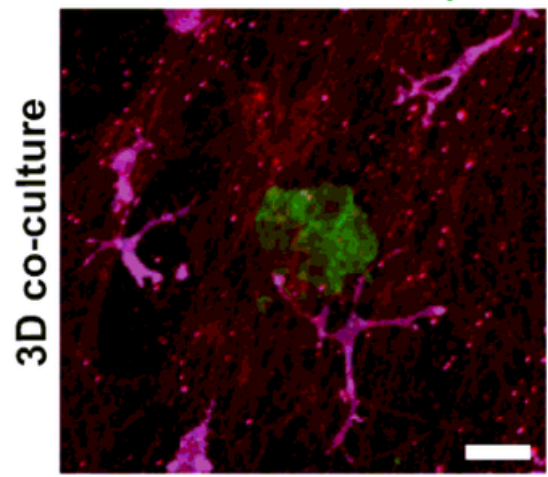

D

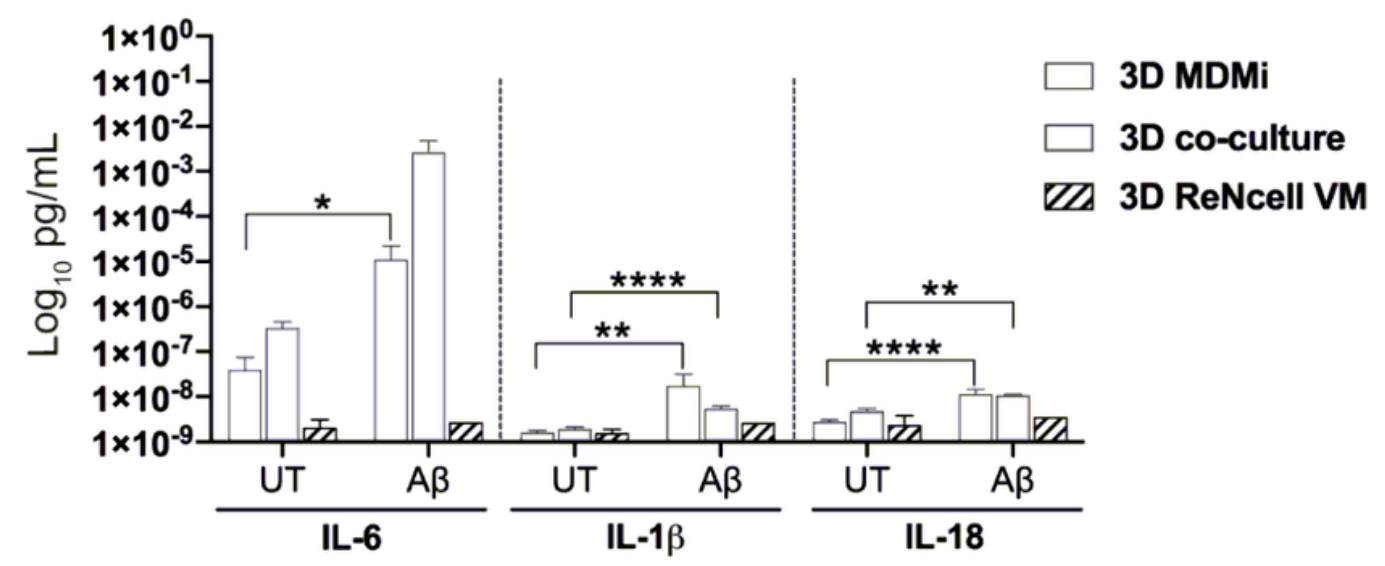

Figure 2

MDMi in 3D co-culture with ReNcell VM-derived neuro-glial cells exhibit inflammatory responses towards $A \beta$ aggregates. (A) Schematic depicting how the 3D co-culture is generated: monocytes pre-mixed with Matrigel are added to a 3D culture of ReNcell VM differentiated for 1 day (left). The co-culture is left to differentiate for 35 days, giving rise to a mixed population of MDMi with ReNcell VM-derived neuro-glial cells (right). (B) Immunofluorescence analysis of MDMi and ReNcell VM 3D co-culture at day 35 of differentiation. MDMi were stained for Iba1 (arrows) and ReNcell VM were stained for the neuron marker 
$\beta 3$-tubulin (TUBB3) and the astrocyte marker GFAP. Scale bars, $100 \mu \mathrm{m}$. (C) Immunofluorescence image of 3D co-cultures containing FITC-A $\beta$ aggregates. Scale bar, $100 \mu \mathrm{m}$. (D) Concentration of secreted proinflammatory cytokines IL-6, IL-1 $\beta$ and IL-18 by 3D MDMi $(n=2)$ and ReNcell VM $(n=1)$ mono-cultures and $3 D$ co-cultures $(n=3)$ upon exposure to FITC-A $\beta$ aggregates. Data are presented as mean \pm SEM. Unpaired Student's t test with or without Welch's correction, two-tailed in J; ${ }^{*} P<0.05, * \star P<0.01, * \star \star * P<$ 0.0001 .

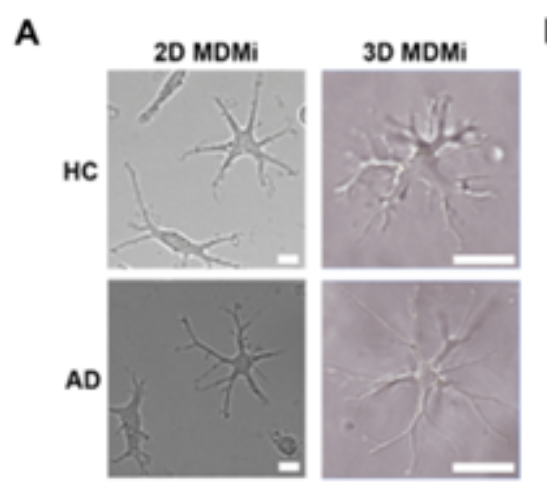

B

\section{C}
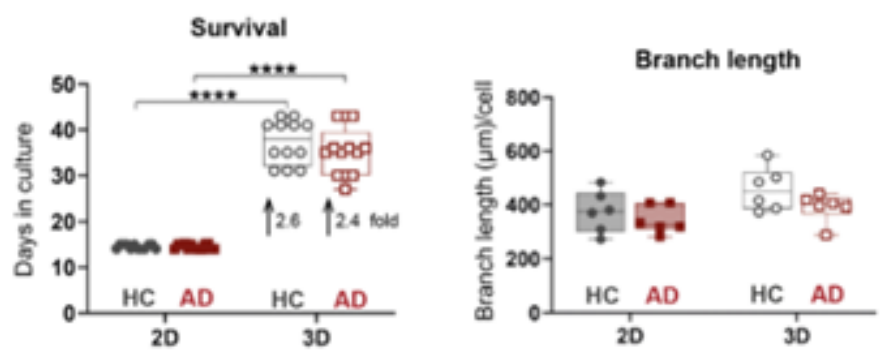

D

E
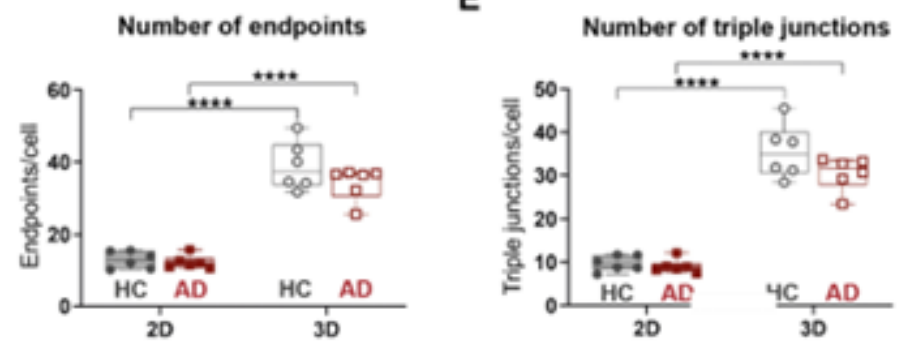

$\mathbf{F}$ Number of quadruple junctions

G

H
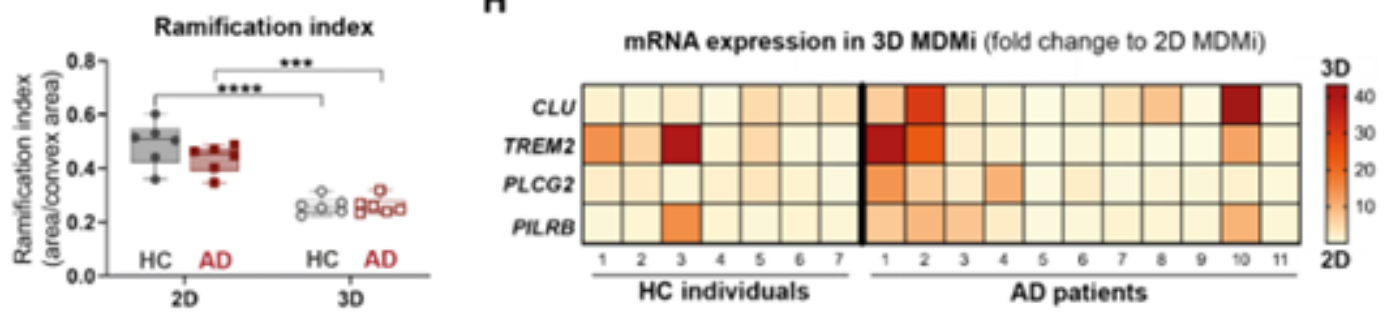

I

$\mathbf{J}$
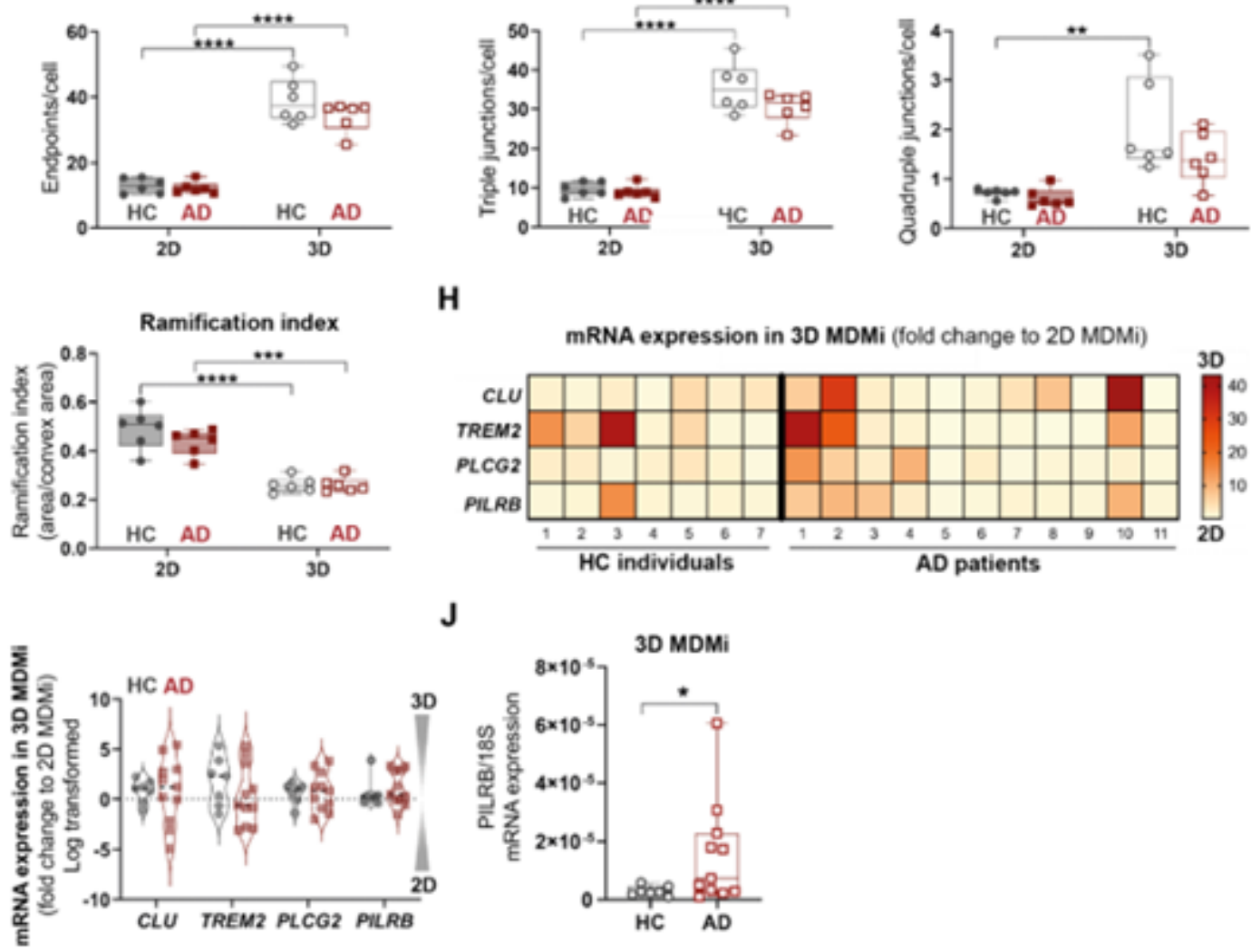

\section{Figure 3}

AD-associated phenotypes in 2D and 3D MDMi mono-cultures related to morphology and expression of $A D$ risk genes. (A) Representative bright field images of $H C$ and $A D M D M i$ in $2 D$ and $3 D$ mono-cultures. Scale bars, $100 \mu \mathrm{m}$. (B) Survival of HC $(n=12)$ and AD $(n=13)$ MDMi in 2D and 3D mono-cultures. Quantification of morphological parameters in 2D and 3D MDMi from both HC $(n=6)$ and $A D(n=6)$ 
cohorts, including (C) branch length, (D) number of endpoints, $(E)$ number of triple junctions, $(F)$ number of quadruple junctions and $(G)$ ramification index. $(H)$ Heatmap representing $H C(n=7)$ and $A D(n=11)$ individual-specific fold changes in gene expression levels of the AD risk genes CLU, TREM2, PLCG2 and PILRB in 3D MDMi compared to 2D. Red-yellow colour spectrum shows relative fold change of 3D MDMi as compared to 2D MDMi. (I) Violin plot representation of fold change (log transformed) of mRNA expression in 3D to $2 D H C(n=7)$ and $A D(n=11)$ MDMi. $(J)$ mRNA expression of the AD risk gene PILRB in 3D HC $(n=7)$ and AD $(n=11)$ MDMi. Data are presented as mean \pm SD. Each single data point represents one biological replicate. Two-way ANOVA with Tukey's multiple comparison test; unpaired

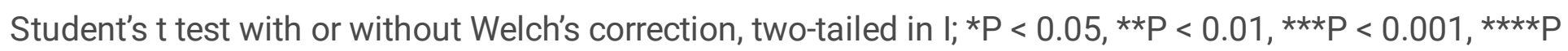
$<0.0001$. 
A

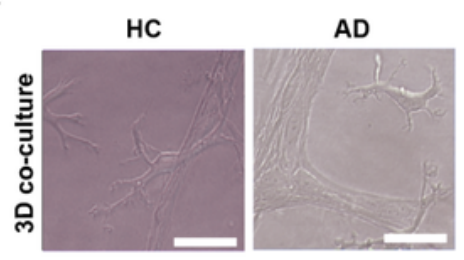

B

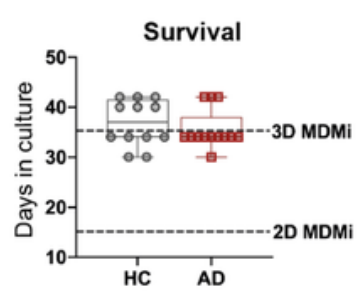

C

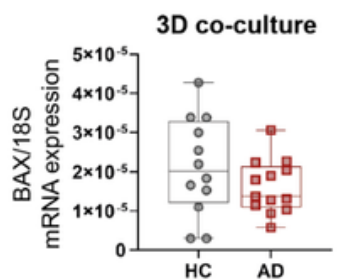

D
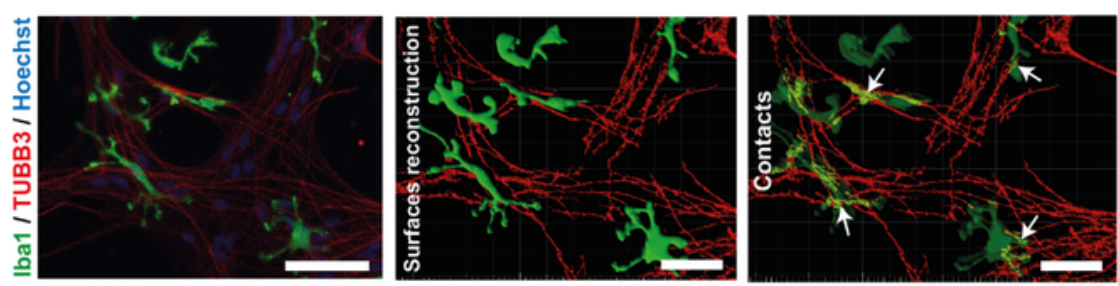

E

F

G

H

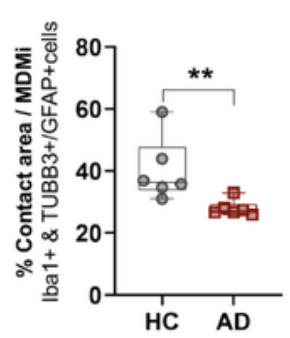

I

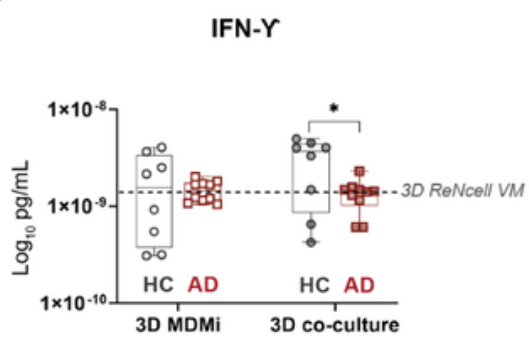

L

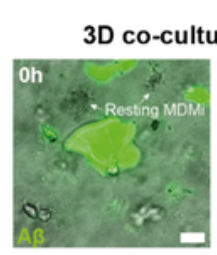

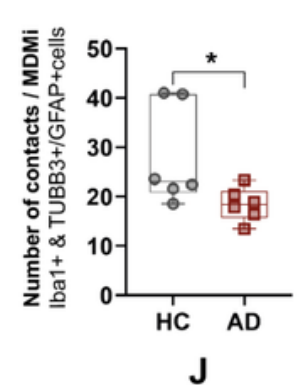

J

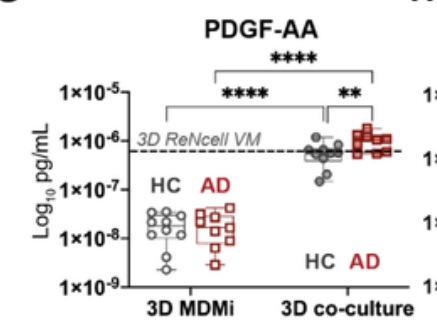

K
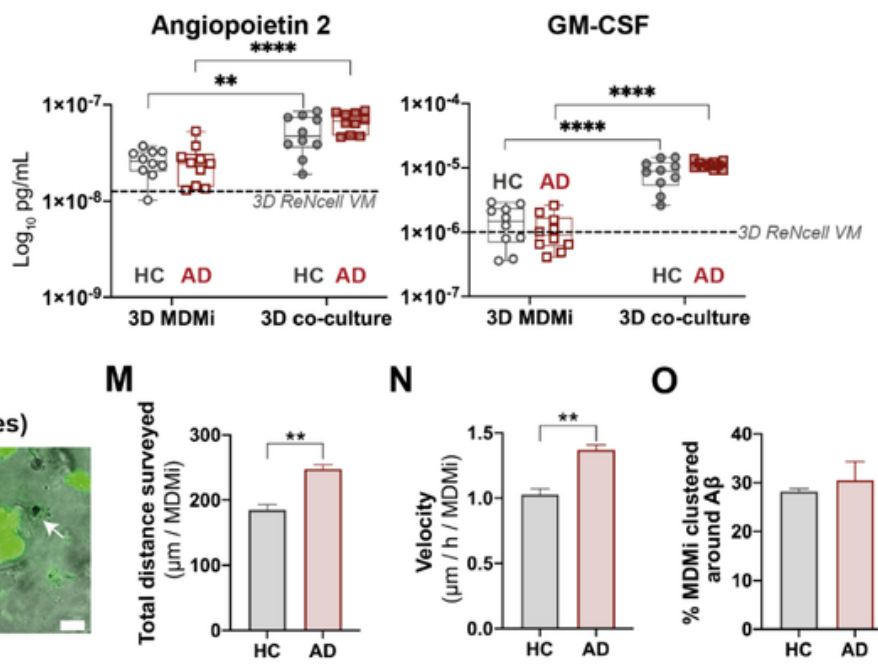

N

0
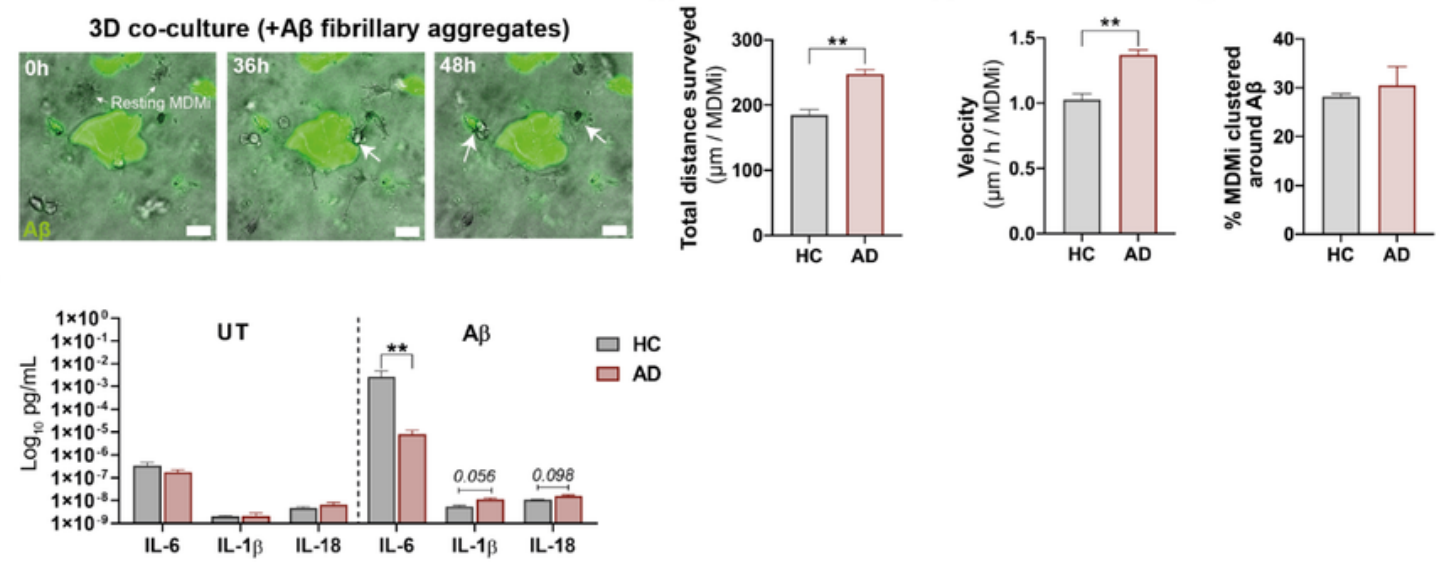

Figure 4

AD-associated phenotypes in 3D co-cultures related to cell-to-cell interaction with ReNcell VM, secretory activity and responses to $A \beta$ aggregates. (A) Representative bright field images of $H C$ and $A D$ co-cultures. Scale bars, $100 \mu \mathrm{m}$. (B) Survival of HC $(n=12)$ and AD $(n=13)$ MDMi in 3D co-cultures. (C) Gene expression of the pro-apoptotic marker BAX in HC $(n=12)$ and $A D(n=13) 3 D$ co-cultures. (D) Immunostaining against lba1 (green) and TUBB3 (red) and 3D surface reconstruction of a 3D co-culture. 
Areas of contact between Iba1+ (MDMi) and TUBB3+ (ReNcell VM) cells are highlighted in yellow (white arrows). Scale bars, $100 \mu \mathrm{m}$. Quantification of (E) contact area and (F) number of contacts between Iba1+ and TUBB3+/GFAP+ cells in HC $(n=6)$ and AD $(n=6) 3 D$ co-cultures. Concentration of $(G)$ plateletderived growth factors AA (PDGF-AA), (H) erythropoietin (EPO), (I) interferon- $\gamma$ (IFN- $-\mathrm{Y}),(\mathrm{J})$ angiopoietin 2 and (K) granulocyte-macrophage colony stimulating factor (GM-CSF) secreted by $\mathrm{HC}(\mathrm{n}=8-10)$ and $\mathrm{AD}$ ( $\mathrm{n}$ = 9-10) MDMi in 3D mono-cultures and co-cultures. Baseline secretion by 3D ReNcell VM mono-cultures is represented with a dotted black line. (L) Representative images of 3D co-cultures containing FITC-A $\beta$ aggregates in which MDMi exhibit a resting, ramified morphology at $\mathrm{Oh}$ and progressively become polarised acquiring an activated, round morphology with enlarged soma upon reaching and clustering on the $A \beta$ deposit at $36 \mathrm{~h}$ and $48 \mathrm{~h}$ (white arrows). Scale bars, $100 \mu \mathrm{m}$. See also Supplementary Movie S3. $(M)$ Total surveillance distance, $(N)$ velocity and $(0)$ clustering around $A \beta$ aggregates of $H C(n=2)$ and $A D(n=4) M D M i$ in $3 D$ co-cultures containing $A \beta$ aggregates. $(P)$ Concentration of pro-inflammatory cytokines IL-6, IL-1 $\beta$ and IL-18 secreted by untreated (UT) and A -treated HC $(n=3)$ and $A D(n=4) M D M i$ in 3D co-cultures. Data are presented as mean \pm SD in B, C, E-K; mean \pm SEM in M-P. Each single data point represents one biological replicate. Unpaired Student's t test with or without Welch's correction, twotailed in B, C, M-P; Mann-Whitney test, two-tailed in E, F; two-way ANOVA with Tukey's multiple comparison test in $\mathrm{G}-\mathrm{K} ; * \mathrm{P}<0.05,{ }^{\star *} \mathrm{P}<0.01, \star \star \star \mathrm{P}<0.001$, $* \star \star \star \mathrm{P}<0.0001$. 
A

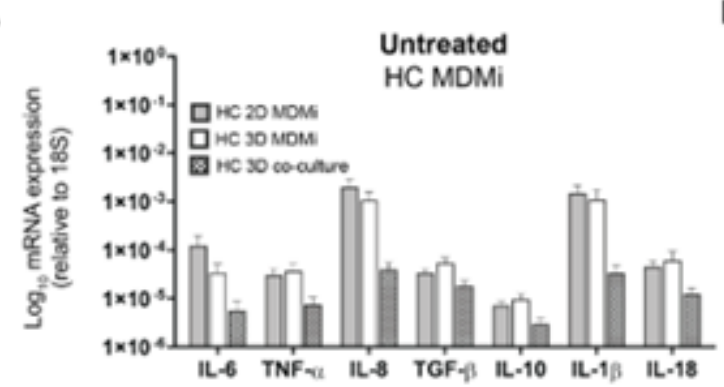

C

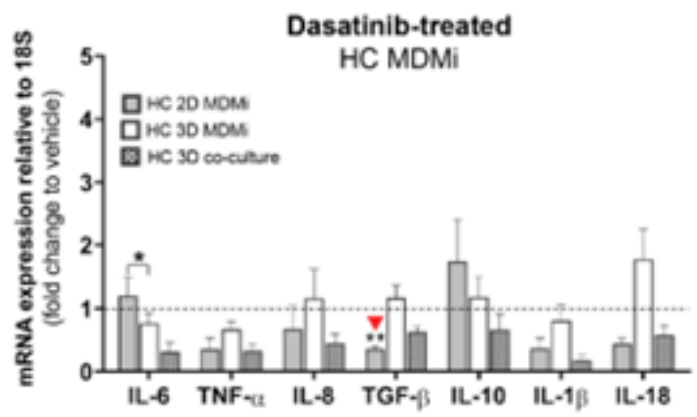

$\mathbf{E}$

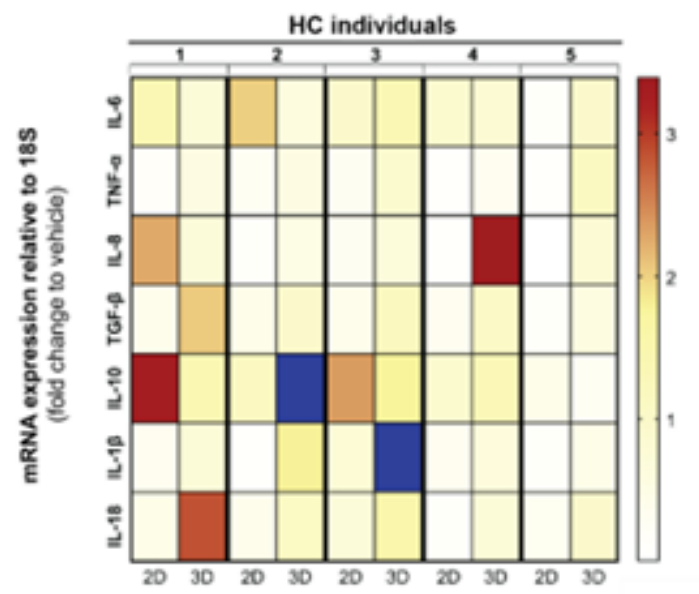

B

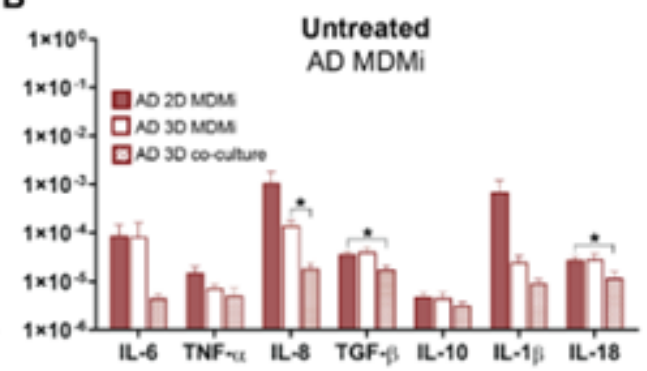

D

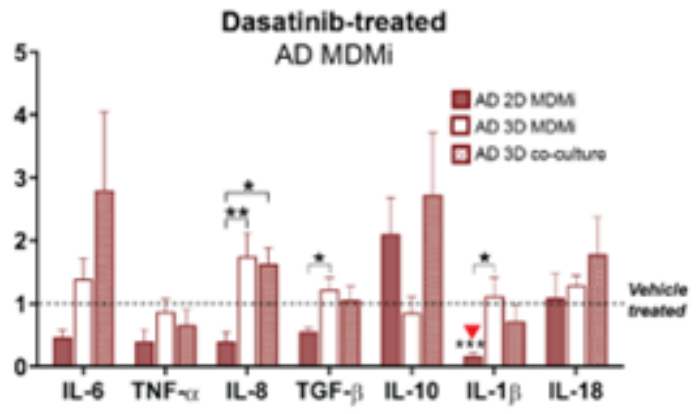

$\mathbf{F}$

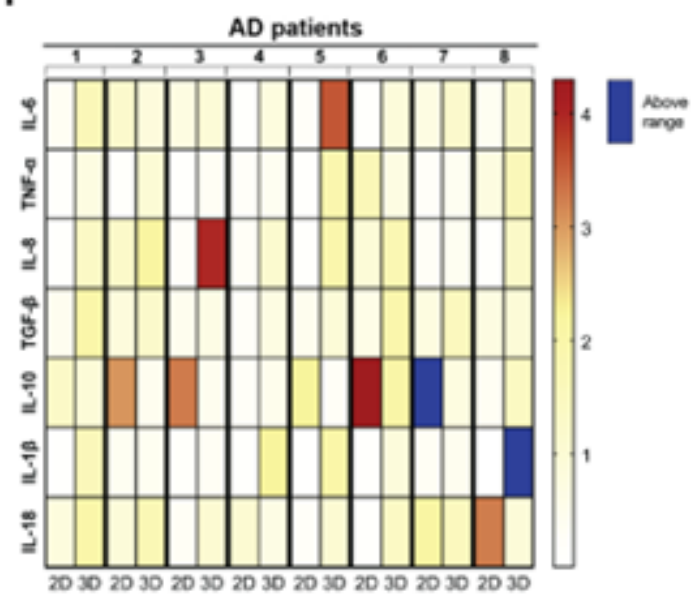

\section{Figure 5}

Dasatinib treatment induces cytokine expression responses that differ between culture format and are heterogeneous among $\mathrm{HC}$ and $\mathrm{AD}$ MDMi. Log-transformed mRNA expression of the inflammatory cytokines IL-6, TNF- $\alpha$, IL-8, TGF- $\beta$, IL-10, IL-1 $\beta$ and IL-18 in untreated (A) HC ( $n=8)$ MDMi and (B) AD ( $n=$ 12) $M D M i$ in all culture formats ( $2 \mathrm{D}$ and $3 \mathrm{D}$ mono-cultures and $3 \mathrm{D}$ co-cultures). Fold change in cytokine mRNA expression levels following $24 \mathrm{~h}$ exposure to $100 \mathrm{nM}$ dasatinib compared to vehicle (DMSO)treated cultures in (C) HC ( $n=6)$ and (D) AD $(n=8)$ 2D and 3D MDMi mono-cultures and 3D co-cultures. Red arrowheads indicate significance compared to vehicle-treated condition. Dotted black lines represent baseline responses of vehicle-treated cultures. Heatmaps showing (E) HC $(n=5)$ and $(F) A D(n=8)$ donors-specific changes in mRNA expression from MDMi mono-cultures in 2D and 3D. Red-yellow colour spectrum represents relative fold change of mRNA expression after dasatinib treatment compared to vehicle. Expression changes falling outside the displayed range are indicated in dark blue. Data are 
presented as mean \pm SEM. One-way ANOVA with Dunnett's multiple comparison test; ${ }^{\star \star P} \mathrm{P}<0.01$, ${ }^{\star \star P}<$ $0.01, * \star * P<0.001$.

\section{Supplementary Files}

This is a list of supplementary files associated with this preprint. Click to download.

- Supplementaryfile1.docx

- Supplementaryfile2.MovieS1.mp4

- Supplementaryfile3.MovieS2.mp4

- Supplementaryfile4.MovieS3.mp4 